\title{
On the excitation of resonant double Kelvin waves in the Barents Sea Opening
}

\author{
Yoshie Kasajima \& Aleksey Marchenko
}

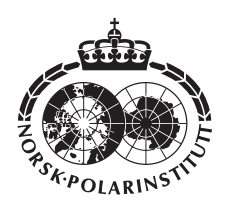

In the northern Barents Sea Opening (BSO) the $\mathrm{K}_{1}$ tidal energy is predominant in the diurnal tidal frequency band, suggesting the generation of a topographic wave with the $\mathrm{K}_{1}$ tidal frequency. Tidal energy of the $\mathrm{K}_{1}$ component becomes strong where bottom topography undulates in the BSO and the scale of the undulation is close to the wavelength of the $\mathrm{K}_{1}$ wave. An analytical model is developed to investigate the energy enhancement mechanism of the tidally induced topographic wave due to a resonance between tidal current, a topographic wave and periodic topography. The wave excited by the resonance is identified as a resonant double Kelvin wave (DKW) and the significant $\mathrm{K}_{1}$ energy in the BSO could be due to the excitation of the resonant DKW.

Y. Kasajima, Norwegian Polar Institute, Polar Environmental Centre, N-9296 Tromsø, Norway. A. Marchenko, General Physics Institute, Russian Academy of Science, 38 Valvilova str. 117942 Moscow, Russia.

Significant tidal energy is often observed when the tide interacts with topography. In the Arctic area, enhanced diurnal tides are observed on the Yermak Plateau (Padman et al. 1992), in the northwestern Barents Sea (Kasajima et al. unpubl. ms), near Bjørnøya (Bear Island) (Huthnance 1981) and on the East Greenland shelf (Lam 1999). Tidal simulations in the Arctic area also exhibit enhanced diurnal tides on the shelf slopes (e.g. Gjevik 1990; Kowalik 1994; Kowalik \& Proshutinsky 1995).

The significant energy enhancement can be explained by a local resonance or an excitation of a topographic wave whose group velocity is close to zero. As shown in the next section, $\mathrm{K}_{1}$ tidal simulations show that tidal vortices appear along the shelf slope in the Barents Sea Opening (BSO). The size of the tidal vortices changes as they proceed northward, due to the variation of the bottom slope, and this variation of the bottom slope makes it possible to generate waves with the major diurnal frequencies, both $\mathrm{K}_{1}$ and $\mathrm{O}_{1}$. However, observations show that the $\mathrm{K}_{1}$ component is enhanced significantly while other diurnal tides are small (Kasajima et al. unpubl. ms). The
BSO is connected to the Storfjord Channel and the Bjørnøya Channel, and the bottom contours undulate in a cross-shelf direction (Fig. 1). The estimated wavelength of the topographic wave with the $\mathrm{K}_{1}$ tidal frequency from the observations in the northern BSO has a scale to the width of the Storfjord Channel. The energy enhancement in only the $\mathrm{K}_{1}$ frequency band seem to be related to the wavelength and the topographic scale.

In this paper, we formulate the mechanism of resonance between a topographic wave, tidal current and periodic topography to explain the enhancement of energy in the $\mathrm{K}_{1}$ frequency in the BSO. The bottom slope is the most important factor for the determination of the wave characteristics. However, here we focus on the effect of the topographic undulation and ignore the effect of the slope. The paper is organized as follows. First, computed surface elevation of the $\mathrm{K}_{1}$ tide in the northern BSO shows the tidal vortices along the BSO. Next, the features of the tidal current are formulated, and in the subsequent section the properties of tidal waves at the depth discontinuity and a double Kelvin wave (DKW) are investigated. We then develop the theory to describe the 


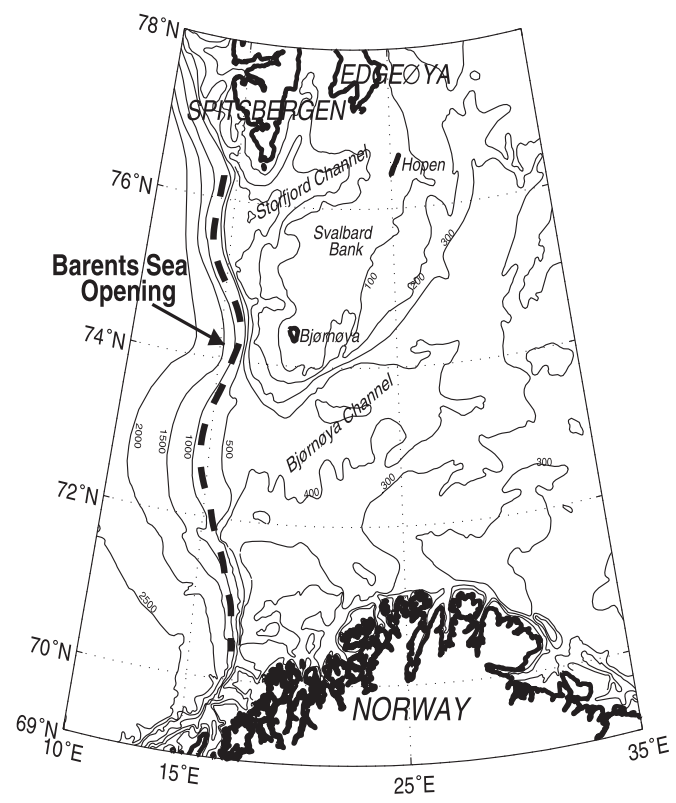

Fig. 1. Topography of the western Barents Sea. The dotted thick line shows the Barents Sea Opening (BSO).

resonant excitation of DKW and, in the final section, discuss the $\mathrm{K}_{1}$ tide in the BSO.

\section{Numerical simulation}

The $\mathrm{K}_{1}$ tide is simulated in the BSO with SINMOD (Slagstad 1987) with a horizontal grid size of $4 \mathrm{~km}$. The open boundary condition is defined by the previously applied model for the Barents Sea with $20 \mathrm{~km}$ horizontal grid size (Kasajima et al. unpubl. ms). The computation area is shown in Fig. 2 and the northern part of the BSO is focused on here.

Figure 3 shows the surface elevation of six phases in the diurnal tidal cycle in the northern part of the BSO. Tidal vortices appear along the BSO slope. They consist of a pair of anticyclonic and cyclonic vortices. Since the sub-inertial topographic wave is a vorticity wave the vortex pair along the slope is a clear signal of the topographic wave. The large vortex on Svalbard Bank, on the other hand, does not consist of a vortex pair, indicating that it is a pulse rather than a continuum wave.

The tidal vortices become clear near Bjørnøya. They are very weak to the south of Bjørnøya, and the large vortex on Svalbard Bank is weakened

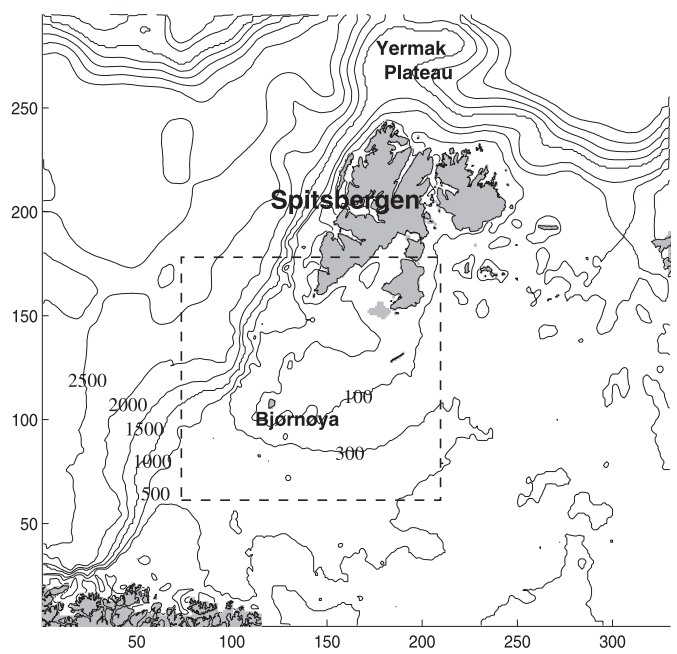

Fig. 2. Computation area of the $4 \mathrm{~km}$ horizontal grid model. The open boundary condition is defined by the larger area of the $20 \mathrm{~km}$ horizontal grid model. The area enclosed by the dotted line is focused on in this paper.

as it approaches Bjørnøya, suggesting that it does not contribute to the vortex formation on the BSO slope. The vortices along the BSO slope are formed locally west of Bjørnøya, where bottom contours turn eastward. The energy flux of the $\mathrm{K}_{1}$ tide averaged over one $\mathrm{K}_{1}$ tidal period along the northern BSO (Fig. 4) supports the formation of vortex near Bjørnøya. Energy flux from Svalbard Bank is nearly zero and it is clear that the energy flux increases in the region where tidal vortex is formed.

The above results suggest that topographic undulation plays an important role in wave generation. In the following sections, we consider the physical mechanism of the excitation of a DKW by the resonance between the tidal current and undulating topography.

\section{Tidal oscillations}

We define non-dimensional variables:

$t=f t^{\prime}, x=\frac{x^{\prime}}{L}, y=\frac{y^{\prime}}{L}, u=\frac{u^{\prime}}{U}, v=\frac{v^{\prime}}{U}, \eta=\frac{\eta^{\prime}}{a}, H=\frac{H^{\prime}}{H_{0}}$,

where $t, x$ and $y$ are the time and horizontal coordinates, $f$ is the Coriolis parameter, $\mathbf{v}=(u, v)$ is depth-averaged horizontal velocity, $\eta$ is the perturbation of the water surface, and $H$ is water depth. The variables with primes denote dimen- 

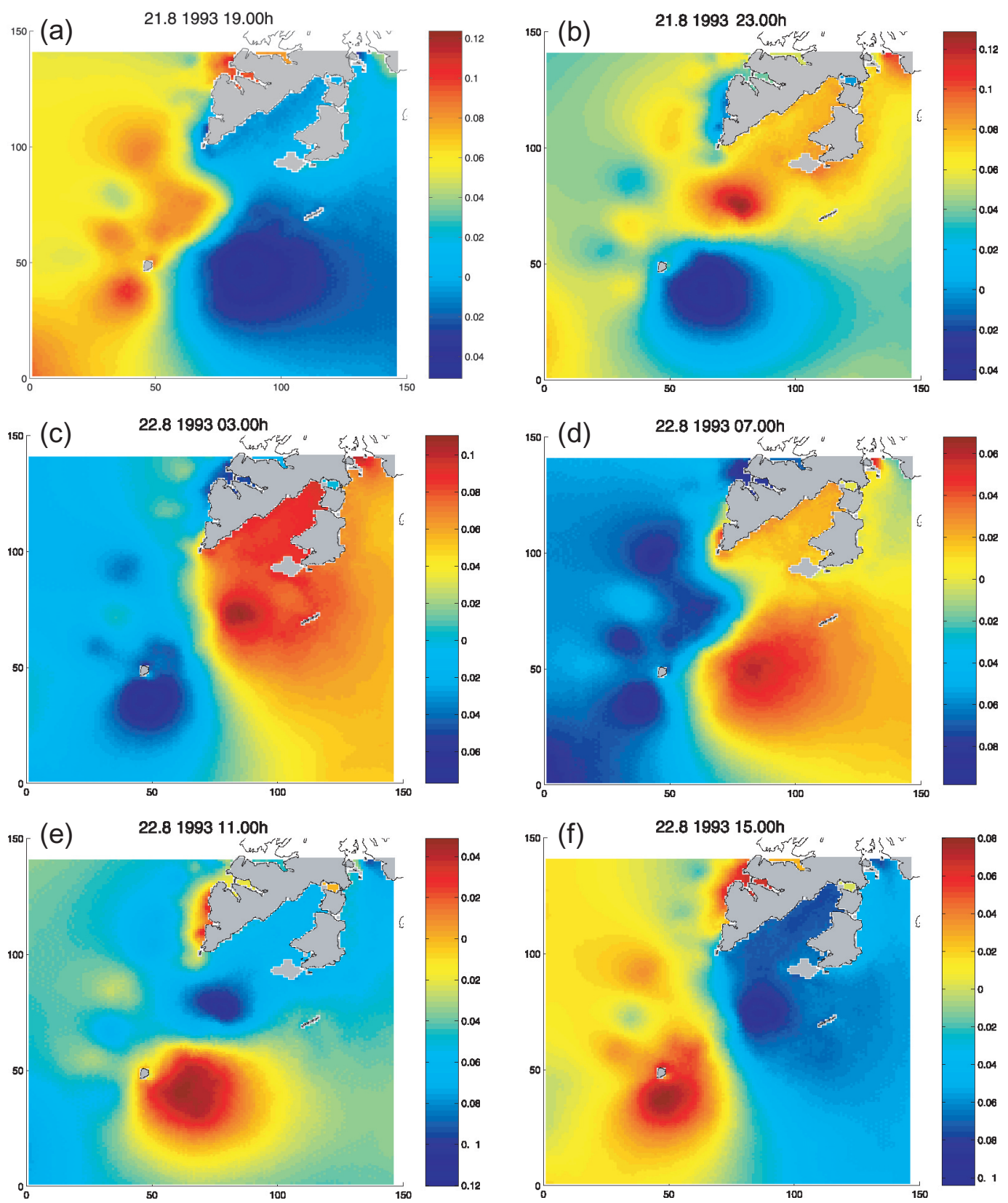

Fig. 3. Surface elevation of the six phases of the $\mathrm{K}_{1}$ tide in the north-western Barents Sea.

sional variables. Typical scales are $f=1.4 \times 10^{-4} \mathrm{~s}^{-1}$, $L=100 \mathrm{~km}, H_{0}=1000 \mathrm{~m}, U=10^{-2} \mathrm{~ms}^{-1}$ and $a=1.0 \mathrm{~m}$. Vertically integrated equations of momentum and mass balance are written in nondimensional form as (1.1):

$$
\frac{\partial u}{\partial t}-v=-\mu \frac{\partial \eta}{\partial x}+\frac{\partial \Omega}{\partial x}
$$

$$
\begin{gathered}
\frac{\partial v}{\partial t}+u=-\mu \frac{\partial \eta}{\partial y}+\frac{\partial \Omega}{\partial y} \\
\frac{\partial \eta}{\partial t}+\frac{\partial(u H)}{\partial x}+\frac{\partial(v H)}{\partial y}=0
\end{gathered}
$$

where $\mu=g H_{0}(f L)^{-2} \approx 50$. Non-dimensional diur- 


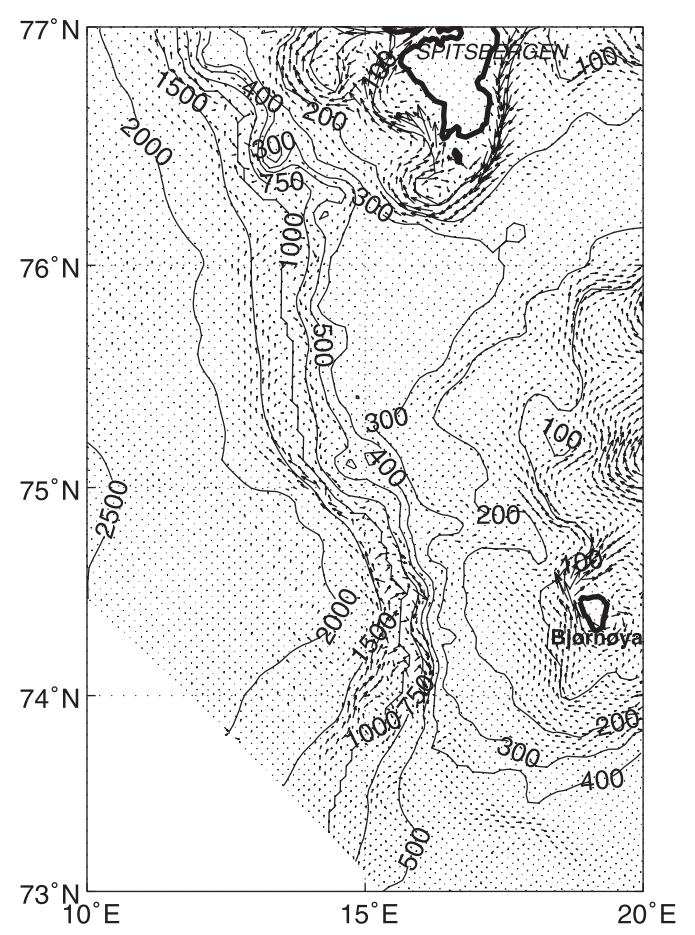

Fig. 4. Dimensionless energy flux averaged over $\mathrm{K}_{1}$ tidal period in the northern BSO area. The south-western part is missing because the database does not cover that area.

nal tidal potential $\Omega$ is defined as:

$$
\Omega=\left(x \Omega_{x}+y \Omega_{y}\right) e^{i \omega_{t} t}+\text { c.c }, \Omega_{x, y}=\text { const }
$$

where c.c. denotes complex conjugation, and $\omega_{t}<1$ is non-dimensional frequency of diurnal tides scaled by the Coriolis parameter $f$. The tidal potential is considered as energy source causing water oscillations. Other energy sources related to incoming waves are not considered in the present paper.

From Equation (1.1) energy conservation takes the form:

$$
\frac{\partial E}{\partial t}=-\mu \nabla \cdot \Pi+\mathrm{A}
$$

where the energy density, $E$, the energy flux, $\Pi$, and the work of the tidal forces, $A$, are defined as:

$$
\begin{gathered}
E=\left(H\left(u^{2}+v^{2}\right)+\mu \eta^{2}\right) / 2, \quad \Pi=\eta \mathbf{v} H, \\
A=H\left(\frac{\partial \Omega}{\partial x} u+\frac{\partial \Omega}{\partial y} v\right) .
\end{gathered}
$$

Assuming that the solution of Equation (1.1) is a periodic function of time, we find the time aver- aged form of (1.2):

$$
\mu \nabla \cdot\langle\Pi\rangle_{t}=\langle A\rangle_{t} .
$$

We denote the time average of a function $G$, by $\langle G\rangle_{t}=T_{t}^{-1} \int_{0}^{T_{t}} G d t$, where $T_{t}=2 \pi \omega_{t}^{-1}$.

The equations (1.1) have a simple solution when $H$ is constant:

$$
\mathbf{v}=\mathbf{V} e^{i \omega_{t} t}+\text { c.c. }, \quad \eta=0,
$$

where the components of the vector $\mathbf{V}$ are defined as:

$$
V_{\boldsymbol{x}}=\frac{i \omega_{t} \Omega_{x}+\Omega_{y}}{1-\omega_{t}^{2}}, \quad V_{y}=\frac{i \omega_{t} \Omega_{y}-\Omega_{x}}{1-\omega_{t}^{2}} .
$$

One can see that the time-averaged energy flux and the work induced by the tidal current (1.4) are zero, $\langle\Pi\rangle_{t}=0,\langle A\rangle_{t}=0$. In the next section we introduce the topographic effect on the tidal current and show the modification of the tidal energy flux. Using the tidal potential makes it possible to formulate the solution of (1.1) in the vicinity of depth discontinuity in simple analytic form.

\section{Interaction of the tidal current with a rectilinear depth discontinuity}

For simplicity of mathematical manipulation, water depth is assumed to be a step structure in the $x$-direction. The depth discontinuity line (DD) is expressed as $x=\varepsilon x_{d}(y)$. The dimensionless water depth is defined by the formulas:

$$
H=1, x<\varepsilon x_{d}(y) ; \quad H=\chi, x>\varepsilon x_{d}(y)
$$

The small dimensionless parameter $\varepsilon \ll 1$ is proportional to the amplitude of the variation of the $\mathrm{DD}$ in the $y$-direction. The DD is rectilinear when $\varepsilon=0$. The conditions of continuity of water mass flux and pressure at the DD (matching conditions) are written as:

$$
\lim _{x \rightarrow x_{d} x^{-0}} \mathbf{v} \cdot \mathbf{n}=\chi \lim _{x \rightarrow x_{d}+0} \mathbf{v} \cdot \mathbf{n}, \lim _{x \rightarrow x_{x^{-}} 0} \eta=\lim _{x \rightarrow x_{x^{+}} 0} \eta,
$$

where $\mathbf{n}$ is the unit vector normal to the DD. For the case without lateral boundaries low frequency motions $\left(\omega_{t}<1\right)$ with a rectilinear DD under the action of the tidal potential are described by the formulas:

$$
\begin{gathered}
\eta=h_{0} \exp \left(i \omega_{t} t+k_{0}^{ \pm} x\right)+\text { c.c. } \\
\mathbf{v}=\left(\mathbf{V}+\mathbf{v}_{0}^{ \pm} \exp \left(k_{0}^{ \pm} x\right)\right) e^{i \omega_{t} t}+\text { c.c. }
\end{gathered}
$$

The constants $h_{0}$ and $\mathbf{v}_{0}^{ \pm}=\left(u_{0}^{ \pm}, v_{0}^{ \pm}\right)$are defined as: 
Fig. 5. (a) The $x$-dependencies of the velocity $v$, and (b) the average energy flux $\left\langle\Pi_{y}\right\rangle_{t}$ induced by the DKW with $\omega=0.5$ and $\chi=0.3$.

Fig. 6. (a) The dependence of sea depth jump $\chi$ over DD from the frequency $\omega$ of the resonant DKW with unity non dimensional wave number. (b) The dependence of the coefficient $K$ from the frequency of the resonant DKW.
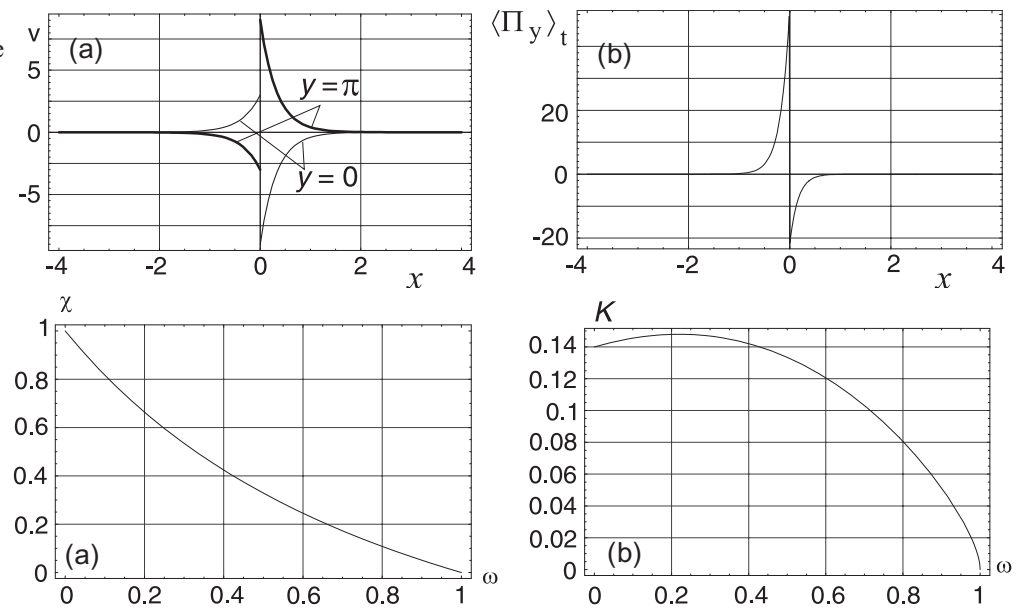

$h_{0}=-i \kappa V_{x}, \quad u_{0}^{ \pm}=-\frac{i \omega_{t} \mu k_{0}^{ \pm}}{1-\omega_{t}^{2}} h_{0}, \quad v_{0}^{ \pm}=\frac{i u_{0}^{ \pm}}{\omega_{t}}$,

where

$\kappa=\frac{(1-\chi)\left(1-\omega_{t}^{2}\right)}{\mu \omega_{t}\left(k_{0}^{-}-\chi k_{0}^{+}\right)}, k_{0}^{+}=-k_{0}^{-} \chi^{-1 / 2}, k_{0}^{-}=\left(\left(1-\omega_{t}^{2}\right) / \mu\right)^{1 / 2}$.

The superscripts "+" and "-" are related to the values of the coefficients in the regions $x>0$ and $x<0$, respectively.

The energy flux of the solution (2.3) averaged over one tidal period is equal to $\langle\Pi\rangle_{t}=\left(0,\left\langle\Pi_{y}\right\rangle_{t}\right)$. The component of energy flux in the $y$-direction, $\left\langle\Pi_{y}\right\rangle_{t}$, is equal to $\Pi_{0}^{-}$when $x<0$, and $\chi \Pi_{0}^{+}$when $x>0$, where

$$
\Pi_{0}^{ \pm}=\kappa e^{k_{0}^{ \pm} x}\left(i V_{x}^{*} V_{y}-i V_{x} V_{y}^{*}+\frac{2 \mu k_{0}^{ \pm}}{1-\omega_{t}^{2}} \kappa V_{x}^{*} V_{x} e^{k_{0}^{ \pm} x}\right),
$$

where the asterisk denotes complex conjugation.

Longuet-Higgins (1968a) has shown that there is another free wave solution with subinertial frequency of the equations (1.1) without tidal potential, satisfying the matching conditions (2.2) with $\varepsilon=0$, i.e. a double Kelvin wave (DKW). A DKW propagates along a DD in the positive $y$-direction and its amplitude decays away from the discontinuity. The frequency, $\omega$, and wave number in the $y$-direction, $k_{y}$, satisfy the dispersion equation:

$$
D\left(\omega, k_{y}, \mu, \chi\right) \equiv \chi\left(\omega k_{-1}^{+}+k_{y}\right)-\omega k_{-1}^{-} k_{y}=0,{ }_{(2.5)}
$$

where $\quad k_{-1}^{+}=-\left(\left(1-\omega^{2}\right) /(\mu \chi)+k_{y}^{2}\right)^{1 / 2}$

and $\quad k_{-i}=\left(\left(1-\omega^{2}\right) / \mu+k_{y}^{2}\right)^{1 / 2}$.

Water surface elevation and velocity field by the DKW are described by the formulas:

$$
\eta=h_{-1} \exp \left(k_{-1}^{ \pm} x+i \theta\right)+\text { c.c, }
$$

$$
\mathbf{v}=\mathbf{v}_{-1} \exp \left(k_{-1}^{ \pm} x+i \theta\right)+\text { c.c. }
$$

where $\theta=\omega t+k_{y} y$. The amplitude, $h_{-1}$, of the DKW is an arbitrary constant, while the components of the vector $\mathbf{v}_{-1}^{ \pm}$are defined by the formulas

$$
u_{-1}^{ \pm}=\frac{-i \mu\left(\omega k_{-1}^{ \pm}+k_{y}\right)}{1-\omega^{2}} h_{-1}, v_{-1}^{ \pm}=\frac{\mu\left(k_{-1}^{ \pm}+\omega k_{y}\right)}{1-\omega^{2}} h_{-1} .
$$

The solution (2.6) has a positive energy flux,

$$
\int_{-\infty}^{\infty}\left\langle\Pi_{y}\right\rangle_{t} d x>0
$$

in the $y$-direction, and there is no energy flux in the $x$-direction, $\left\langle\Pi_{x}\right\rangle_{t}=0$. The dependencies of $\left\langle\Pi_{y}\right\rangle_{t}$ and $v$ on $x$ are shown in Fig. 5a, b. One can see that the solutions (2.3) and (2.6) are independent. Further, we show that an interaction of tidal currents (2.3) and DKWs is possible and has resonant properties when $x_{d}=\sin y$.

\section{Resonant excitation of a DKW in the vi- cinity of a periodic depth discontinuity}

We consider the interaction of a tidal current with a periodic DD and a DKW as a resonant interaction of three waves (Kaup 1976), the frequencies and wave numbers of which satisfy the conditions $\omega_{1}+\omega_{2}=\omega_{3}$ and $k_{1}+k_{2}=k_{3}$. In our case, the first wave is the tidal current, so we set $\omega_{1}=\omega_{t}$ and $k_{l}=0$. The second wave is the wave of the bottom topography, the frequency of which is $\omega_{2}=0$ and the wave number of which is $k_{2}=-1$. The third wave is a DKW, the frequency and the wave number of which are $\omega_{3}=\omega_{t}$ and $k_{3}=-1$.

The condition of the resonant interaction has 


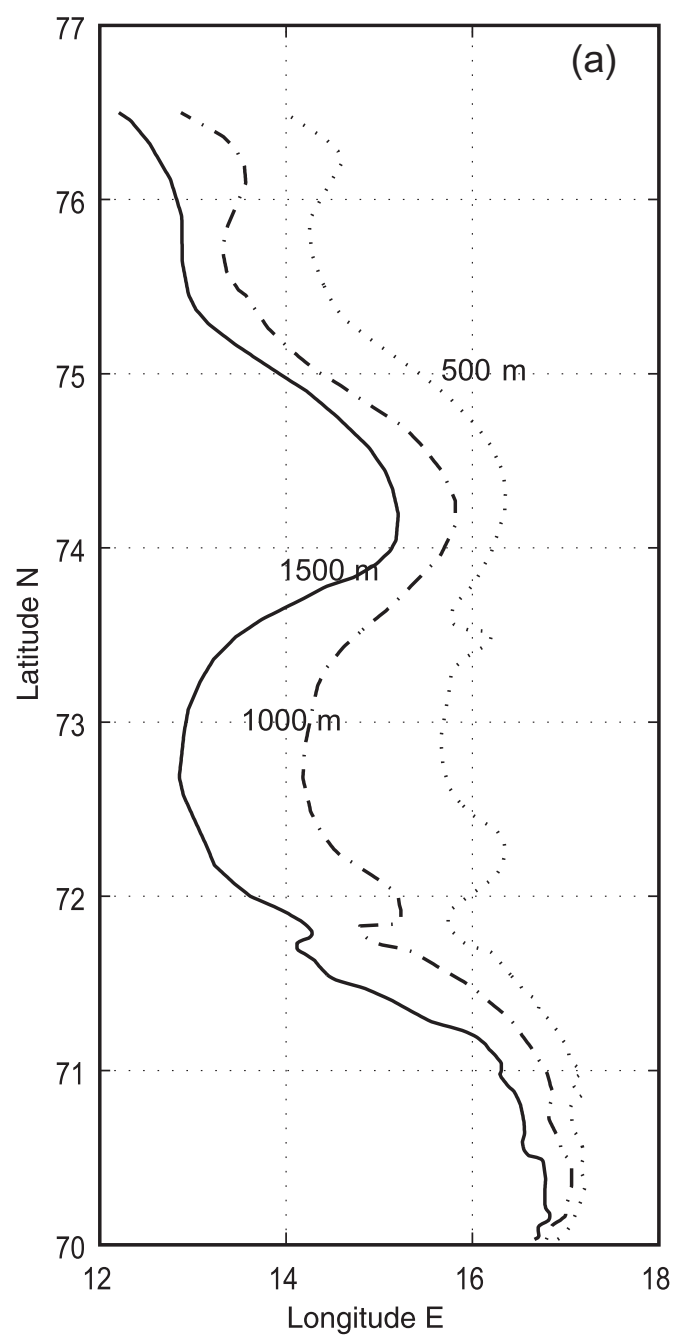

the form:

$$
\omega=\omega_{t}, k_{y}=-1 .
$$

In other words, the tidal frequency $\omega_{t}$ and unity wave number characterizing the wavelength of the DD line are the roots of the dispersion equation (2.5) for the DKW. We name the condition (3.1) as dimensionless resonant condition, which is the condition for the excitation of a DKW due to the interaction of a tidal current of frequency $\omega_{t}$ with a periodic depth discontinuity, the space period of which is characterized by unity wave number. We name the DKW satisfying condition (3.1) the resonant DKW.

To derive the amplitude equation for the resonant DKW we replace the DD line from $x=\varepsilon \sin$ $y$ to $x=0$ in the matching condition (2.2),

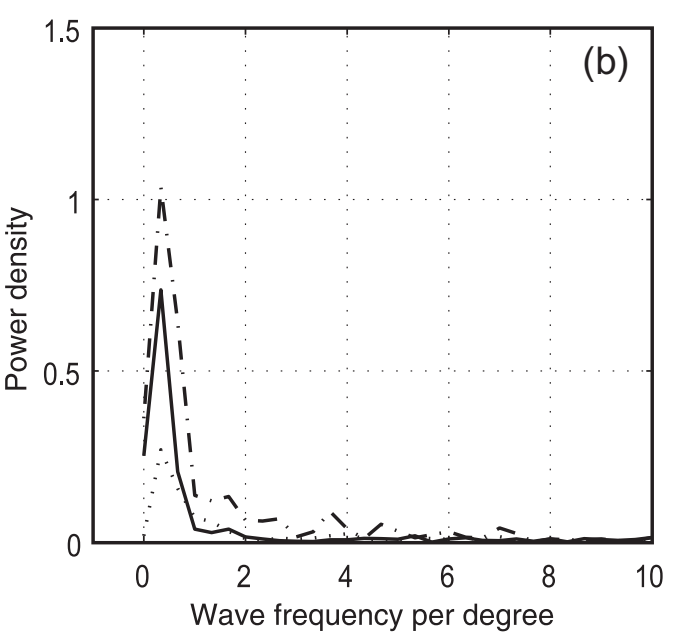

Fig. 7. (a) Bottom contours of 500 (dotted line), 1000 (dashed line) and 1500 (solid line) $\mathrm{m}$ in the BSO, and (b) the spectra of these bottom contours.

$$
\lim _{x \rightarrow-0} \Theta_{1}=\chi \cdot \lim _{x \rightarrow+0} \Theta_{1}, \quad \lim _{x \rightarrow-0} \Theta_{2}=\lim _{x \rightarrow+0} \Theta_{2}
$$

where $\Theta_{1}=u+\varepsilon \frac{\partial u}{\partial x} \sin y-\varepsilon v \cos y+O\left(\varepsilon^{2}\right)$,

$$
\Theta_{2}=\eta+\varepsilon \frac{\partial \eta}{\partial x} \sin y+O\left(\varepsilon^{2}\right)
$$

Following the method of multiscale expansions (Whitham 1974; Hsieh \& Mysak 1980), we find the solution of equations (1.1) satisfying the conditions (3.2) in the form of asymptotic expansions in the small parameter $\varepsilon$ and chose the amplitudes of the wave modes to be slow functions of time $T=\varepsilon t$ :

$$
\begin{gathered}
\mathbf{v}=e^{i \omega_{t} t}\left(\mathbf{V}+\sum_{n=-\infty}^{\infty} \mathbf{v}_{n}(T, x) e^{i n y}\right)+\text { c.c. }, \\
\eta=e^{i \omega_{t} t} \sum_{n=-\infty}^{\infty} \eta_{n}(T, x) e^{i n y}+\text { c.c. }
\end{gathered}
$$

To find the amplitude equation describing the time variation of the complex amplitude $h_{-1}=\left.\eta_{-1}^{ \pm}\right|_{x=0}$ of the resonant DKW we use the technique developed for the description of parametric excitation of edge waves in the vicinity of a crack in floating ice (Marchenko 1999). In this approach the amplitude equations follow from the matching conditions, and equations of momentum and mass balance (1.1) are used to define the behaviour of the solution in the direction normal to the DD (see appendix).

Note that in contrast to a typical three wave interaction, the amplitudes of the first and the second waves, which are proportional to $V_{x}$ and $\varepsilon$, are given. Therefore, we have derived only one 


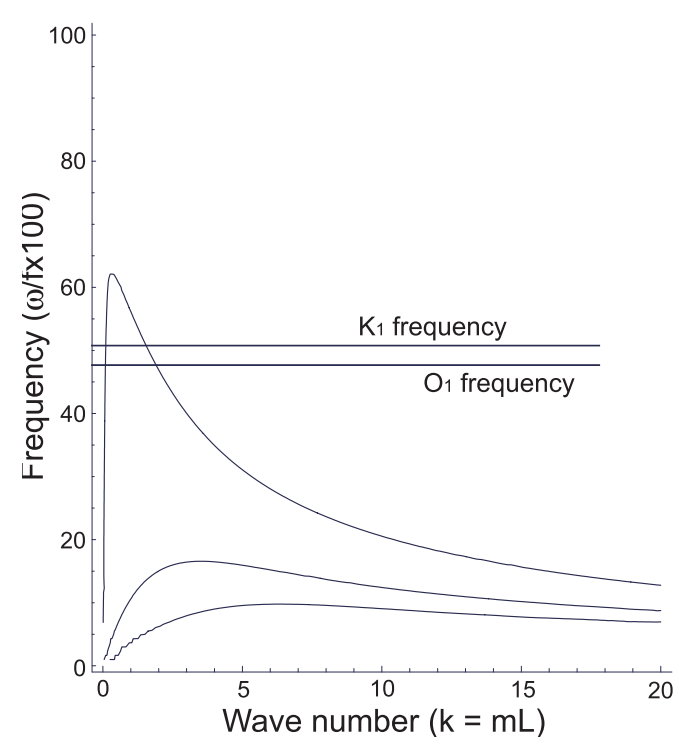

Fig. 8. Dispersion relation of the DKW in the northern BSO.

amplitude equation for the complex amplitude $h_{-1}$ of the resonant DKW.

The amplitude equation in the vicinity of the resonance $(\Delta=\mathrm{O}(\varepsilon))$ has the form:

$$
\frac{\partial h_{-1}}{\partial T}+\frac{i \Delta}{\varepsilon} h_{-1}=K h_{0} .
$$

The constants $\Delta$ and $K$ are defined in the appendix. One can see that the DKW amplitude $h_{-1}$ tends to infinity when $\Delta \rightarrow 0$ if we set $\partial h_{-1} / \partial T=0$. Equation (3.4) describes the rise of the resonant DKW until its energy becomes comparable with the forcing wave defined by the formulas (2.3). The typical non-dimensional time of this process is $\tau=K^{-1}$, and the typical dimensional time is $T^{\prime}=(\varepsilon f K)^{-1}$.

The dependence $\chi=\chi(\omega)$ following from the dispersion equation (2.5) at $k_{y}=-1$ and $\mu=50$ is shown in Fig. 6a. The dependence $K=K(\omega)$ at $k_{y}=-1, \mu=50$ is shown in Fig. 6b. One can see that $\chi \approx 0.3$ and $K \approx 0.13$ when $\omega=0.5$. Assuming $\varepsilon=0.1$ one finds the typical time $T^{\prime}=6.4$ days. Thus the typical time of the excitation of the DKW is close to several days.

From (3.4) it follows that the absolute value of the amplitude, $\left|h_{-1}\right|$, of the resonant DKW is proportional to the time with the coefficient $\varepsilon\left|K h_{0}\right|$. The value of $\left|h_{0}\right|$ is proportional to the modulus of the velocity $\left|V_{x}\right|$ of the tidal current in the direction normal to the DD and to the amplitude $\varepsilon$ of the variations of the DD line. Thus, the spatial orientation of the DD line influences the rate of the excitation of the resonant DKW.

The slow spatial variation of the DKW is described by the equation:

$$
\frac{\partial h_{-1}}{\partial T}-c_{g} \frac{\partial h_{-1}}{\partial Y}+\frac{i \Delta}{\varepsilon} h_{-1}=K h_{0},
$$

where $c_{g}$ is group velocity of the DKW and $Y=$ $\varepsilon y$ is a slow spatial variable along the DD line. This equation is reduced to (3.4) in the sliding frame reference running with group velocity $c_{g}$. Evidently the rise of $h_{-1}$ during time $\tau$ will occur over the distance $c_{g} \tau$. Therefore, the most interesting application of the theory is related to the case when the group velocity of the resonant DKW is small.

\section{Discussion}

Using linearized shallow water equations without bottom friction we have shown that the amplitude of the DKW is increased in time due to the interaction of the diurnal tidal current with an unbounded periodic depth discontinuity when the resonant condition (3.1) is satisfied. In reality, bottom friction and nonlinear phenomena restrict the amplitude of the DKW, and the effect of the shelf slope must be considered.

Figure 7a shows the bottom contours with 500, 1000 and $1500 \mathrm{~m}$ depths at the BSO slope. To quantify the wavelength of the bottom contours, spectra are calculated (Fig. 7b) and the dominant wavelength is about $330 \mathrm{~km}$. A simplified topography was produced based on the actual sea bottom profile along $76^{\circ} \mathrm{N}$. We used this simplified topography to derive a dispersion relation of the DKW following Longuet-Higgins (1968b). This is shown in Fig. 8. The $K_{1}$ frequency has two intersects in the first mode and the wavelengths are estimated as $5024 \mathrm{~km}$ and $334 \mathrm{~km}$. At $76^{\circ} \mathrm{N}$ the diameter of the vortex increases approximately to $150 \mathrm{~km}$ in the simulations (Fig. 3 ), then the latter agrees with the horizontal scale of the simulated wave. In addition, the $\mathrm{O}_{1}$ frequency also has intersects with the first mode at the wavelength of $5644 \mathrm{~km}$ and $264 \mathrm{~km}$ but the short wavelength is smaller than the scale of topography. Therefore, it is possible that the significant $\mathrm{K}_{1}$ tidal energy is due to the excitation of the resonant DKW. 
Acknowledgements.-Discussion with H. Svendsen and Z. Kowalik gave us new insight into the problem under study. We thank the referees for their help in improving this paper. This work has been supported partially by the Norwegian Research Council under Project Code 128087/730.

\section{Appendix: Derivation of the ampli- tude equations}

Substituting expansions (3.3) into equations (1.1) and setting the coefficients of the exponentials $\exp ($ iny) to be equal, one finds the system of three equations that defines $u_{n}, v_{n}$, and $\eta_{n}$ for each $n$. Each such system includes terms of the order $\mathrm{O}(\varepsilon)$ which are proportional to the first derivatives by slow time $T$. Using the method of successive approximations, one finds the solution with accuracy $\mathrm{O}(\varepsilon)$ to be:

$$
\begin{gathered}
u_{n}^{ \pm}=\left(\alpha_{u, n}^{ \pm} h_{n}^{ \pm}+\varepsilon\left(\beta_{u, n}^{ \pm} x+\gamma_{u, n}^{ \pm}\right) \frac{\partial h_{n}^{ \pm}}{\partial T}\right) e^{k_{n}^{ \pm} x}, \\
v_{n}^{ \pm}=\left(\alpha_{v, n}^{ \pm} h_{n}+\varepsilon\left(\beta_{v, n}^{ \pm} x+\gamma_{v, n}^{ \pm}\right) \frac{\partial h_{n}^{ \pm}}{\partial T}\right) e^{k_{n}^{ \pm} x}, \\
\eta_{n}^{ \pm}=\left(h_{n}^{ \pm}+\varepsilon \beta_{n}^{ \pm} x \frac{\partial h_{n}^{ \pm}}{\partial T}\right) e^{k_{n}^{ \pm} x},
\end{gathered}
$$

where the superscripts plus and minus are related to the regions $\mathrm{x}>0$ and $\mathrm{x}<0$, respectively. Coefficients $\alpha, \beta$ and $\gamma$ are defined by the formulas:

$$
\begin{gathered}
\alpha_{u, n}^{ \pm}=\alpha_{u}, \quad \alpha_{v, n}^{ \pm}=\alpha_{v}, \quad \beta_{u, n}^{ \pm}=\beta_{u}, \beta_{v, n}^{ \pm}=\beta_{v}, \\
\beta_{n}^{ \pm}=\beta, \gamma_{u, n}^{ \pm}=\gamma_{u}, \quad \gamma_{v, n}^{ \pm}=\gamma_{v},
\end{gathered}
$$

where

$$
\begin{array}{cl}
\alpha_{u}=-i \frac{\mu\left(\omega k_{x}+k_{y}\right)}{1-\omega^{2}}, & \alpha_{v}=\frac{\mu\left(k_{x}+\omega k_{y}\right)}{1-\omega^{2}}, \\
\beta_{u}=\beta \alpha_{u}, & \beta_{v}=\beta \alpha_{v},
\end{array}
$$

$\beta=-\frac{i\left(1-\omega^{2}\right)}{2 \mu \omega k_{x} H}\left(1-k_{x} H\left(i \omega \alpha_{u}+\alpha_{v}\right)+i k_{y} H\left(i \omega \alpha_{v}+\alpha_{u}\right)\right)$,

and $\gamma_{u}=-\frac{i \omega \mu \beta+i \omega \alpha_{u}+\alpha_{v}}{1-\omega^{2}}, \gamma_{v}=\frac{\mu \beta+i \omega \alpha_{v}+\alpha_{u}}{1-\omega^{2}}-$

The superscripts plus and minus and subscript $n$ are related to the values of $k_{x}=k_{x, n}{ }^{ \pm}, k_{y}=n$ and $H=H^{ \pm}$, where

$$
k_{x, n}^{ \pm}=\mp \sqrt{\frac{1-\overline{\omega^{2}}}{\mu H^{ \pm}}+n^{2}}, H^{+}=\chi, H^{-}=1 .
$$

Let us substitute equations (3.3) and (I) into the matching conditions (3.2) and set the coefficients of the exponentials $\exp ($ iny) to be equal. For $n=0$ one finds:

$$
h_{0}^{ \pm}=h_{0}+\mathrm{O}(\varepsilon)
$$

where $h_{0}$ is defined by (2.3). From the second matching condition (3.2) for $n=-1$ and formula
(II) it follows that:

$$
h_{-1}^{+}=h_{-1}^{-}-\frac{i \varepsilon}{2}\left(k_{x, 0}^{+}-k_{x, 0}^{+}\right) h_{0}+\mathrm{O}\left(\varepsilon^{2}\right)
$$

Using the first matching condition (3.1) and formulas (I) and (III) one finds:

$$
\frac{\partial h_{-1}}{\partial T}+\frac{i \Delta}{\varepsilon} h_{-1}=K h_{0},
$$

where

$$
\begin{gathered}
h_{-1}=h_{-1}^{ \pm}+\mathrm{O}(\varepsilon), \\
\Delta=\frac{\mu D(\omega,-1, \mu, \chi)}{\left(1-\omega^{2}\right)\left(\chi \gamma_{u,-1}^{+}-\gamma_{u,-1}^{-}\right)}, \\
K=\frac{i\left(\chi \alpha_{u, 0}^{+} k_{x, 0}^{+}-\alpha_{u, 0}^{-} k_{x, 0}^{-}\right)-\chi \alpha_{i, 0}^{+}+\alpha_{i, 0}^{-}+i \chi \alpha_{u,-1}^{+}\left(k_{x, 0}^{+}-k_{x, 0}^{-}\right)}{2\left(\chi \gamma_{u,-1}^{+}-\gamma_{u,-1}^{-}\right)},
\end{gathered}
$$

and $D(\omega,-1, \mu, \chi)$ is defined by (2.5).

\section{References}

Gjevik, B. 1990: Model simulations of tides and shelf waves along the shelves of the Norwegian-Greenland-Barents Sea. Model. Mar. Sys. 1, 187-219.

Hsieh, W. W. \& Mysak, L. A. 1980: Resonant interactions between shelf waves, with applications to the Oregon Shelf. J. Phys. Oceanogr. 10, 1729-1741.

Huthnance, J. M. 1981: Large tidal currents near Bear Island and related tidal energy losses from the North Atlantic. Deep-Sea Res. 28A, 51-70.

Kasajima, Y., Svendsen, H. \& Slagstad, D. unpubl. ms: Topographic waves with the $\mathrm{K}_{1}$ tidal frequency in the Barents Sea.

Kaup, D. J. 1976: The three wave interaction-a nondispersive phenomenon. Stud. Appl. Math. 55, 9-44.

Kowalik, Z. 1994: Modeling of topographically amplified diurnal tides in the Nordic seas. J. Phys. Oceanogr. 24, $1717-1731$.

Kowalik, Z. \& Proshutinsky, A. Y. 1995: Topographic enhancement of tidal motion in the western Barents Sea. $J$. Geophys. Res. 100(C2), 2613-2637.

Lam, F.-P. 1999: Shelf waves with diurnal tidal frequency at the Greenland shelf edge. Deep-Sea Res. Part I 46, 859-923.

Longuet-Higgins, M. S. 1968a: On the trapping of waves along a discontinuity of depth in a rotating ocean. J. Fluid Mech. 31, Part 3, 417-434.

Longuet-Higgins, M. S. 1968b: Double Kelvin waves with continuous depth profiles. J. Fluid Mech. 34, Part 1, 49-80.

Marchenko, A. V. 1999: Parametric excitation of flexuralgravity edge waves in the fluid beneath an elastic ice sheet with a crack. Eur. J. Mech. B-Fluids 18, 511-525.

Padman, L., Plueddemann, A. J., Muench, R. D \& Pinkel, R. 1992: Diurnal tides near the Yermak Plateau. J. Geophys. Res. 97(C8), 12639-12652.

Slagstad, D. 1987: A 4-dimensional physical model of the Barents Sea. SINTEF Rep. STF48 F87013. Trondheim.

Whitham, G. B. 1974: Linear and nonlinear waves. New York: Wiley. 